\title{
Secretome of Mesenchymal Stem Cells Grown in Hypoxia Accelerates Wound Healing and Vessel Formation In Vitro
}

\section{Sushma Bartaula-Brevik*, Anne Isine Bolstad, Kamal Mustafa and Torbjorn O Pedersen}

Department of Clinical Dentistry, University of Bergen, Norway

${ }^{*}$ Corresponding author: Sushma Bartaula-Brevik, Department of Clinical Dentistry, University of Bergen, Arstadveien 19, 5009 Bergen, Norway, E-mail: sushma.bartaula@uib.no

\begin{abstract}
Hypoxia is one of the factors that trigger the release of inflammatory and vasculogenic cytokines during tissue regeneration. Mesenchymal stem cells (MSC) with and without endothelial cells (EC) were cultured in vitro in normoxic and hypoxic environments. The mRNA expression of inflammatory and vasculogenic cytokines were evaluated at $1,12,24$ and 48 hours. After 48 hours of incubation in normoxic and hypoxic conditions, supernatants termed as conditioned medium (CM) from each group were collected and analyzed. The protein level of VEGF-A in the CM was determined by ELISA. The effects of the CM from different groups on EC were evaluated using wound healing- and tube formation assays. The mRNA expression of IL-1 $\beta$, IL- 6 and IL- 8 was up-regulated in both the normoxic and hypoxic co-culture (MSC/EC) group, compared with the mono-culture normoxic group. The VEGF-A protein level was higher in the hypoxic mono- and co-culture group. Wound closure was accelerated by CM from the mono- and co-culture hypoxic groups compared with both normoxic groups. Measurements of tube formation were higher in the hypoxic mono-culture group compared with the normoxic group. The conditioned medium obtained from hypoxia preconditioning of the cells accelerated wound healing and vessel formation in vitro.
\end{abstract}

\section{Keywords}

Mesenchymal stem cells, Endothelial cells, Co-culture, Hypoxia, Angiogenesis, Wound healing

\footnotetext{
Abbreviations

EC: human umbilical vein endothelial cells; MSC: primary human bone marrow derived multipotent stromal cells mesenchymal stem cells; ASC: adipose stem cells; MSCGM: mesenchymal stem cell growth medium; EGM: endothelial cell growth medium; FBS: fetal bovine serum; EGF: epidermal growth factor; FGF: fibroblast growth factor; VEGF: vascular endothelial growth factor; ELISA: enzyme linked immunosorbent assay; CM: conditioned medium;
}

IL: interleukin; ANG: angiopoietin; PDGF: platelet derived growth factor; CXCL: chemokine (C-X-C Motif) ligand; HIF: hypoxia inducible factor; KGF: keratinocyte growth factor; IGF: insulin growth factor; EPO: erythropoietin; TPO: thrombopoietin

\section{Introduction}

The ability of stem cells to self-renew and differentiate into mature cells of different lineages is regulated by both intrinsic programming and extrinsic input from the stem cell niche or microenvironment [1]. The poor post-implantation survival of transplanted cells limit therapeutic efficacy [2]. Several strategies have been postulated to overcome this challenge, which includes preconditioning of the cells by heat shock, oxidative stress and hypoxia [3]. The oxygen concentration in different tissues and organs varies from 2-9\%, whereas bone marrow niches have a lower oxygen concentration at about $1 \%$ [4]. This suggests that bone marrow stem cells could favor a hypoxic microenvironment. Several studies have shown that hypoxia induces release of chemokines, cytokines and growth factors involved in cell proliferation, differentiation, migration, apoptosis and angiogenesis $[5,6]$. However, the effect of shortand long-term hypoxic environments on survival, proliferation and differentiation of mesenchymal stem cells (MSC) is still controversial [7]. It has been shown that by changing the culture conditions, MSC can be directed towards the endothelial cell lineage $[8,9]$. Also, the paracrine effect of implanted MSC promotes vascularization by in growth of host microvasculature into tissue-engineered constructs [10].

\section{Clinged} International Library

Citation: Bartaula-Brevik S, Bolstad Al, Mustafa K, Pedersen TO (2017) Secretome of Mesenchymal Stem Cells Grown in Hypoxia Accelerates Wound Healing and Vessel Formation In Vitro. Int J Stem Cell Res Ther 3:045. doi.org/10.23937/2469-570X/1410045

Received: November 03, 2016: Accepted: February 17, 2017 : Published: February 20, 2017 Copyright: () 2017 Bartaula-Brevik S, et al. This is an open-access article distributed under the terms of the Creative Commons Attribution License, which permits unrestricted use, distribution, and reproduction in any medium, provided the original author and source are credited. 
Different strategies have been suggested to improve vascularization in tissue engineering, which include both pre-vascularized and pre-conditioned constructs. Endothelial cells (EC) have been co-cultured with different cell types, including MSC, adipose stem cells (ASC) and osteoblasts, all with the aim of improving vascularization after being implanted in vivo. It has been shown that the microvasculature in pre-vascularized constructs can interconnect with the host microvasculature, in order to ensure implant survival [11]. Pre-conditioned tissueengineered constructs can alsobegenerated by incorporating different growth factors, or by changing the physical and chemical properties of the scaffold material $[12,13]$. EC were cultured in the conditioned medium obtained from MSC under hypoxic condition, which demonstrated higher angiogenic potential of EC in vitro $[14,15]$.

Pre-vascularization of tissue-engineered construct by directly co-culturing MSC and EC in vitro resulted in microvascular network formation in vivo [16]. Despite of extensive work on co-culture systems, limited effort has been made to address the effect of different culture condition on direct co-culture. The aim of this study was to evaluate how hypoxia influenced MSC grown in mono- and co-culture, and the effect of MSC's secretome on wound healing and vessel formation.

\section{Material and Methods}

\section{Cell culture}

Primary human bone marrow derived MSC and $\mathrm{Hu}$ man umbilical vein endothelial cells were purchased from Lonza (Walkersville, Maryland, United States). MSC were expanded in Mesenchymal Stem Cell Growth Medium (MSCGM ${ }^{\mathrm{rx}}$ ) SingleQuots ${ }^{\mathrm{rx}}$ (Lonza) containing mesenchymal stem cell basal medium supplemented with mesenchymal cell growth supplement (MCGS), L-glutamine and GA-1000 (Gentamicin, Amphotericin-B). EC were expanded in Endothelial Cell Growth Medium-2 (EGM-2 ${ }^{\circ}$ BulletKit (Lonza) containing Endothelial Cell Basal Medium and supplemented with Fetal bovine serum (FBS), human epidermal growth factor (hEGF), hydrocortisone, GA-1000, vascular endothelial growth factor (VEGF), human fibroblast growth factor (hFGF-B), insulin-like growth factor ( $\mathrm{R}^{3}$-IGF-1), ascorbic acid and heparin. Both cell types were expanded at 37 ${ }^{\circ} \mathrm{C}$ and $5 \% \mathrm{CO}_{2}$. Cells older than passage four were not used for the experiments.

\section{Experimental setup}

The cells were seeded in 6-well plates with a seeding density of 200,000 cells/well either MSC alone or MSC/ EC in a 5:1 ratio. The cells were allowed to attach overnight. The next day those two groups were further sub-divided into hypoxic and normoxic groups. The four groups were MSC-NOR (NMSC), MSC/EC-NOR (NMSC/EC), MSC-HYP (HMSC) and MSC/EC-HYP (HMSC/EC). A Modular Incubator Chamber (MIC-101) was used as a hypoxia chamber and the chamber was
Table 1: List of Taqman $®$ probes used for real time reverse transcription polymerase chain reaction analysis.

\begin{tabular}{|l|l|}
\hline Gene symbol & Assay ID \\
\hline GAPDH & Hs99999905_m1 \\
\hline IL-1 $\beta$ & Hs01555410_m1 \\
\hline IL-6 & Hs00985639_m1 \\
\hline IL-8 & Hs00174103_m1 \\
\hline IL-10 & Hs00961622_m1 \\
\hline VEGF-A & Hs00900055_m1 \\
\hline PDGF & Hs00966526_m1 \\
\hline FGF & Hs00266645_m1 \\
\hline ANG-1 & Hs00375822_m1 \\
\hline ANG-2 & Hs01048042_m1 \\
\hline CXCL12 & Hs00171022_m1 \\
\hline
\end{tabular}

filled with a tri-gas mixture of $1 \% \mathrm{O}_{2}, 5 \% \mathrm{CO}_{2}$ and $94 \%$ $\mathrm{N}_{2}$. After 1, 12, 24 and 48 hours the cells were harvested. The cells were trypsinized, and EC were depleted with CD31 Endothelial Cell Dynabeads ${ }^{\circledR}$ (Invitrogen, Carlsbad, California, United States) according to the manufacturer's instructions. The isolated MSC were stored at $-80^{\circ} \mathrm{C}$ before RNA isolation. The medium from each group was collected in aliquots, centrifuged and stored at $-80^{\circ} \mathrm{C}$ for further analysis.

\section{Real-time reverse transcription polymerase chain reaction (RT-PCR)}

Total RNA isolation was done using Maxwell ${ }^{\circledR} 16 \mathrm{LEV}$ simply RNA purification kits (Promega, Madison, Wisconsin, United States). RNA purity and quantification were determined with a Nanodrop Spectrophotometer (Thermo Scientific Nano-Drop Technologies, Wilmington, Delaware, USA). 1000 ng of RNA was reverse transcribed to cDNA using a cDNA kit (Applied Biosystems, Carlsbad, California, United States). Real-time RT-PCR was performed on a StepOne ${ }^{\mathrm{TM}}$ real time RT-PCR system (Applied Biosystems). cDNA corresponding to 10 ng mRNA in each reaction was prepared in duplicates for each target gene and the real-time RT-PCR was run under standard cycling conditions. Different angiogenic and inflammatory genes were evaluated (Table 1).

\section{Enzyme linked immunosorbent assay}

For protein analysis, a commercially available human VEGF-A enzyme-linked immunosorbent assay (ELISA) (R\&D Systems, Minneapolis, Minnesota, United States) was used. Cell culture supernatants from the 48 hours' time point were thawed and the VEGF-A concentration was determined according to the manufacturer's instructions. Optical densities were determined using a FLUOstar OPTIMA microplate reader (BMG LABTECH, Ortenberg, Germany). The values were compared to a known standard curve and the final concentrations are presented in $\mathrm{pg} / \mathrm{ml}$.

\section{Wound healing assay}

Human umbilical vein endothelial cells were cultured in Culture-Insert 24 (80241, ibidi, Martinsried, Germany) at a concentration of 30,000 cells/well in duplicate. The 
culture inserts were carefully removed after the cells reached confluency. A wound of approximately 500 $\mu \mathrm{m}$ width was created by the insert, and the wounded monolayer of cells was washed three times with phosphate buffered saline (PBS) to remove dead cells and debris. The cells were incubated with conditioned medium from MSC-NOR, MSC/EC-NOR, MSC-HYP, MSC/EC-HYP and positive (EGM-2) and negative (MSCGM) control media for 24 hours. Images were taken at the beginning as a baseline, and at 3,6, 12, 18 and 24 hours' time points during the cell migration to close the wound. The images were taken with a Nikon Eclipse Ti microscope (Nikon, Tokyo, Japan) at magnification of $4 \mathrm{x}$ in the phase contrast mode. Images obtained from each group were analyzed with NIS elements AR 3.2 software (NIS elements, Tokyo, Japan). The surface area covered by the wound was measured and exported to a Microsoft Excel (Microsoft Corporation, Redmond, Washington, United States) file before statistical analysis was performed. All the images were normalized with the baseline data.

\section{Tube formation assay}

Growth Factor Reduced Matrigel (BD Biosciences, San Jose, California, United States) was thawed overnight at $4{ }^{\circ} \mathrm{C}, 40 \mu \mathrm{l}$ per well was added to the growth surface
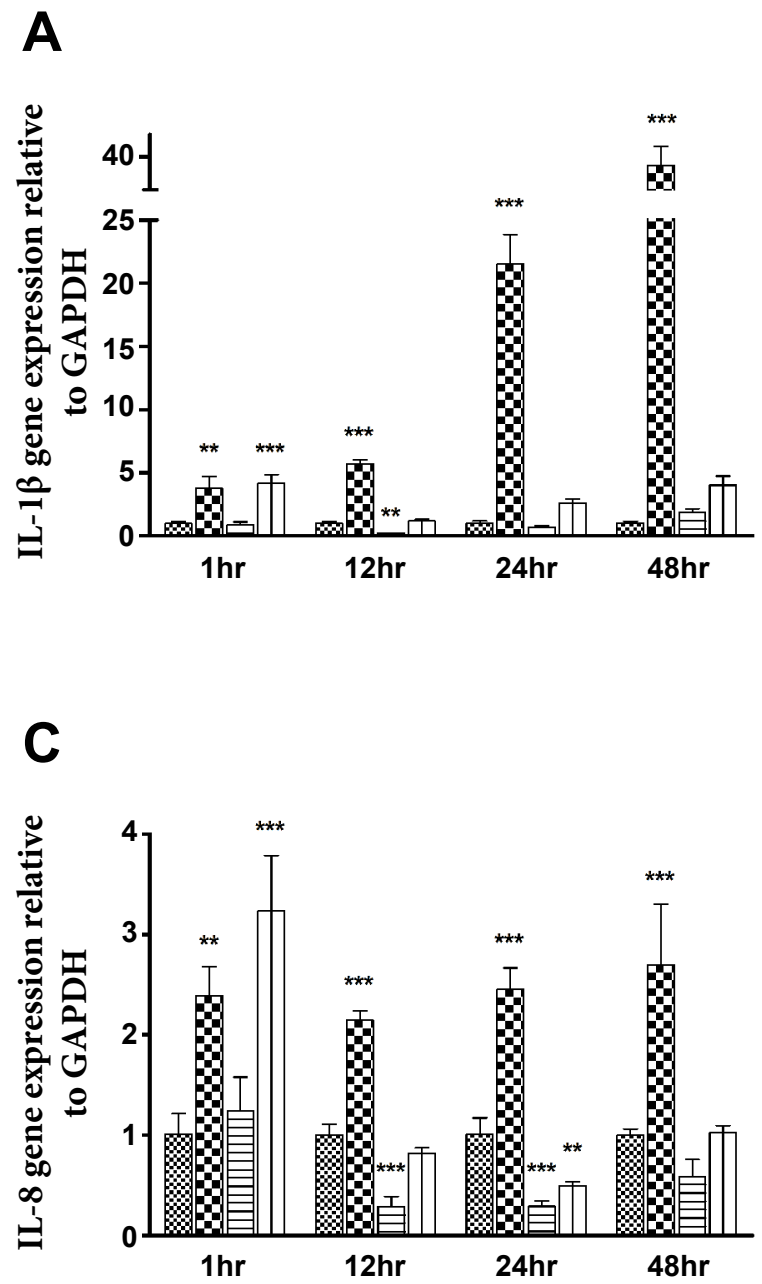

of 96 well plates and incubated for $30 \mathrm{~min}$ at $37^{\circ} \mathrm{C}$ to allow the matrigel to solidify. Human umbilical vein endothelial cells were suspended in conditioned medium from MSC-NOR, MSC/EC-NOR, MSC-HYP, MSC/ EC-HYP and positive (EGM-2) and negative (MSCGM) control media. The cells were seeded onto the solidified matrigel at a density of 20000 cells/well. After incubating for 6 hours, the formation of tube like structures was observed under the microscope. The images were taken with a Nikon Eclipse Ti microscope (Nikon) at magnification of $4 \mathrm{x}$ in the phase contrast mode. The tube formation was quantified using the ImageJ software program (NIH, Bethesda, Maryland, United States) with the Angiogenesis Analyzer plugin [17]. The parameters of tube formation were normalized with control medium (MSCGM).

\section{Results}

\section{Inflammatory and angiogenic gene expression}

The mRNA expression of pro-inflammatory cytokines Interleukin-1 $\beta$ (IL-1 $\beta$ ), Interleukin-6 (IL-6) and Interleukin-8 (IL-8) were evaluated in normoxic and hypoxic culture conditions for 1, 12, 24 and 48 hours. In normoxia, the expression of IL- $1 \beta$ was exponentially

\section{B}
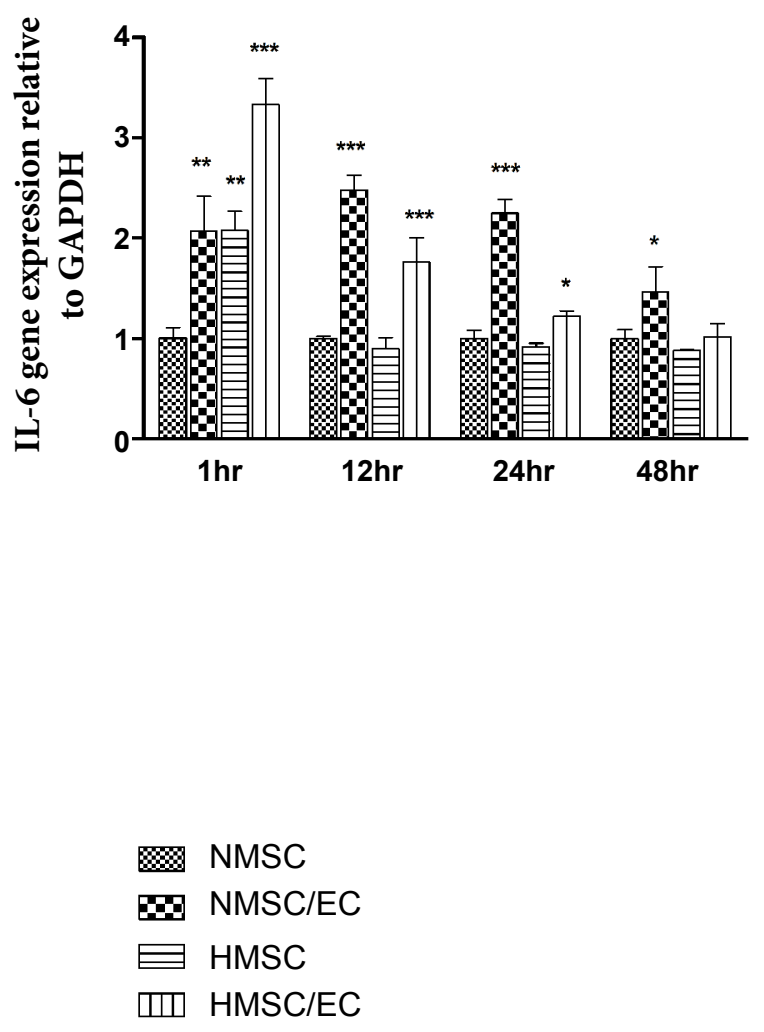

Figure 1: Relative gene expression of inflammatory cytokines: IL-1 $\beta$ (A) IL-6 (B) and IL-8 (C) comparing NMSC/EC, HMSC and HMSC/EC groups with NMSC at different time points. Data are presented as means \pm standard deviation $(n=6)$. ${ }^{*}=p$ $<0.05 ;{ }^{* *}=p<0.01 ;{ }^{* *}=p<0.001$. 
higher in the co-culture group compared with the mono-culture group, whereas in hypoxia, a significant increase was noticed in co-culture group compared with NMSC only at $1 \mathrm{hr}$ and no significant difference between the groups could be found after 12 hours (Figure 1A). IL-1 $\beta$ expression was down-regulated in HMSC group compared with NMSC group at 12 hours. The mRNA expression of IL- 6 was significantly higher in the co-culture normoxic and both hypoxic groups at 1hour compared with NMSC (Figure 1B). HMSC showed no significant difference after 12 hours and HMSC/EC showed no significant difference after 24 hours, although the expression of IL-6 was still significantly higher in NMSC/EC until 28 hours (Figure 1B). The mRNA expression of IL-8 was significantly higher in NMSC/EC and HMSC/EC after 1hour compared to NMSC. At 12 hours, the expression of IL-8 started to reduce significantly in the HMSC group. In the NMSC/EC group, IL-8 showed continuous up-regulation until 48 hours. However, IL-8 expression was lower in HMSC/EC group at 24 hours compared to the NMSC group (Figure 1C). Overall, these results show that the co-culture with EC increased mRNA expression of pro-inflammatory cytokines. Anti-inflammatory cytokine Interleukin-10 (IL-10) was also evaluated but was not expressed by any of the groups.

The mRNA level of VEGF-A was significantly higher expressed in the hypoxic group. The results also showed that the expression of VEGF-A depend on a hypoxic environment, independent of co-seeding with EC (Figure 2A). The mRNA expression of angiopoietin-1 (ANG1) was down-regulated in all the groups compared with the normoxic mono-culture group (Figure 2C), whereas angiopoietin-2 (ANG-2) expression was highly upregulated in the co-culture group irrespective of culture condition (Figure 2D).

The mRNA expression of platelet derived growth factor (PDGF) was down-regulated in the HMSC group at 1 and 12 hours compared with the NMSC group (Figure $3 \mathrm{~A}$ ). The mRNA expression of fibroblast growth factor (FGF) was higher in HMSC at 1 hour, but the expression was down-regulated after 12 hours. The NMSC/EC group
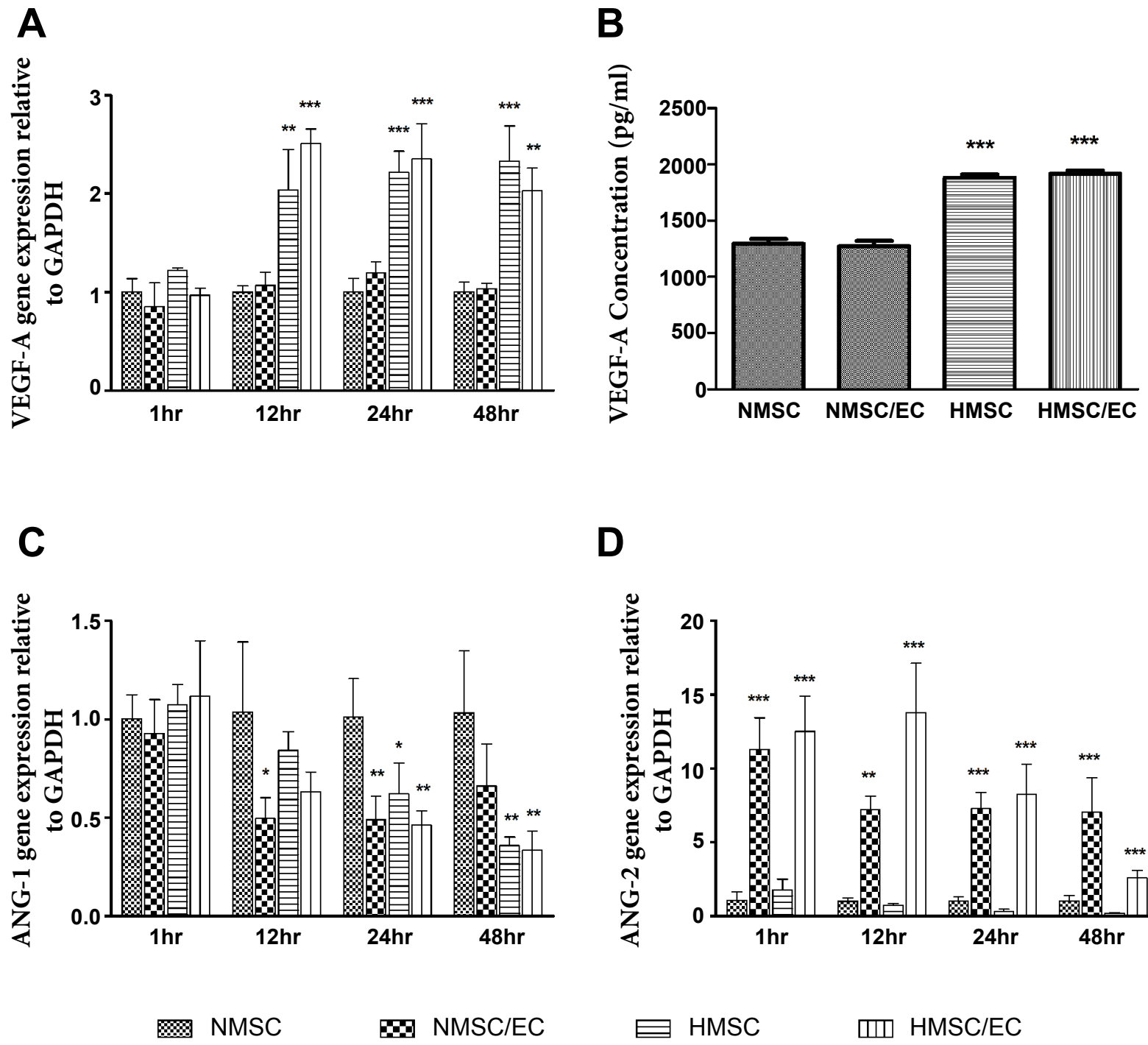

D

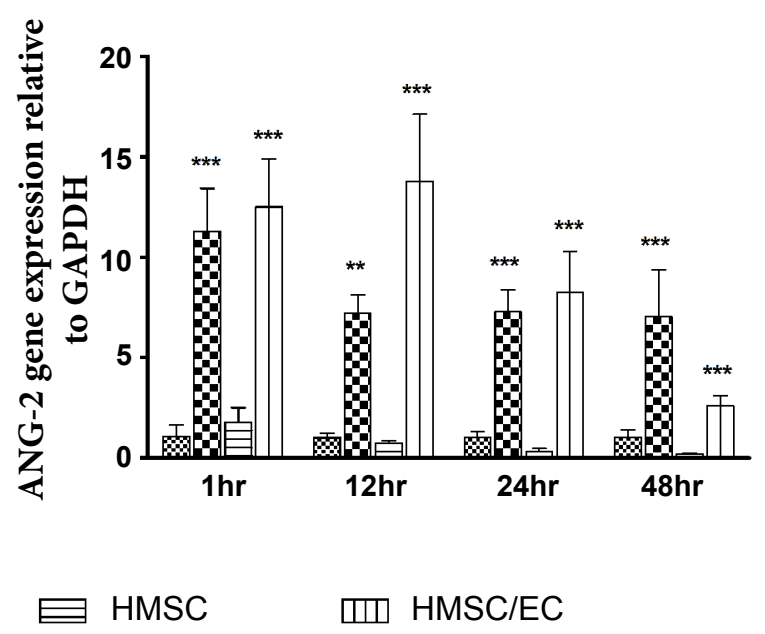

Figure 2: Relative gene expression of VEGF-A (A) ANG-1 (C) and ANG-2 (D) comparing NMSC/EC, HMSC and HMSC/EC groups with NMSC at different time points. Protein expression of VEGF-A as determined by ELISA (B) data are presented as means \pm standard deviation. ${ }^{*}=p<0.05 ;{ }^{* *}=p<0.01 ;{ }^{* *}=p<0.001$. 
A

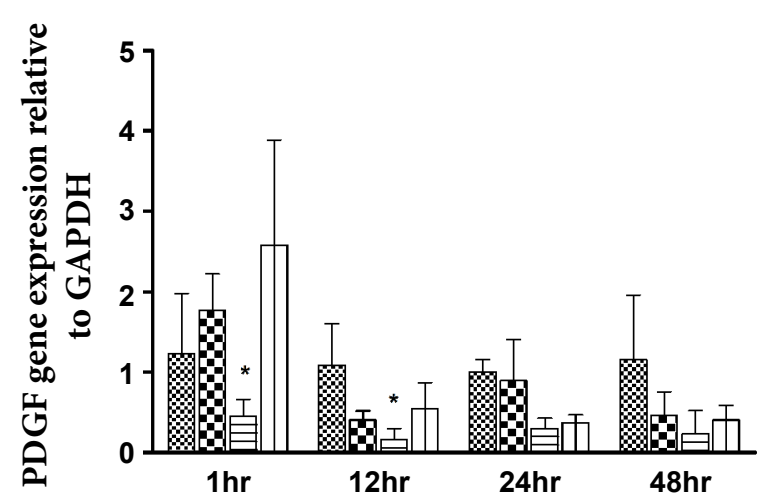

C
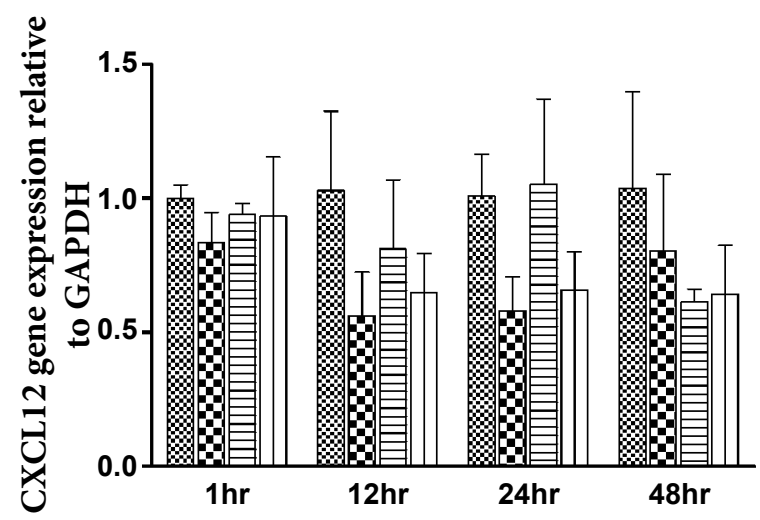

B

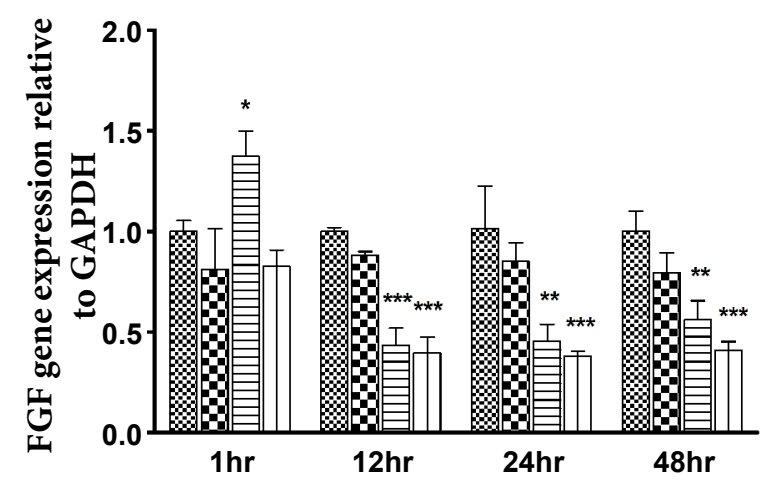

Figure 3: Relative gene expression of PDGF (A) FGF (B) and CXCL12 (C) comparing NMSC/EC, HMSC and HMSC/EC groups with NMSC at different time points. Data are presented as means \pm standard deviation. ${ }^{*}=p<0.05 ;{ }^{* *}=p<0.01 ;{ }^{* * *}$ $=p<0.001$.

showed no significant difference compared to NMSC. The HMSC/EC group showed significant down-regulation after 12 hours compared with the NMSC (Figure $3 \mathrm{~B})$. There was no significant difference in the mRNA expression of CXCL-12 between the groups (Figure 3C).

\section{Elisa}

The protein expression of VEGF-A was evaluated for mono and co-culture groups with and without hypoxia (Figure 2B). VEGF-A expression was higher in the hypoxic group compared with the normoxic group and was statistically significant. Also, the VEGF-A expression on the protein level was independent of the co-culture with EC.

\section{Wound healing assay}

Cell migration towards the wounded area at different time intervals was compared with both positive (EGM2) and negative (CTRL) controls (Figure 4A). The bar diagram comparing the experimental groups to the NMSC group, show that the wound healing was affected by hypoxia and significantly higher in HMSC and HMSC/EC groups respectively (Figure 4B, Figure 4C, Figure 4D and Figure 4E). After 18 hours of incubation, the wound area was completely closed in both hypoxic

\section{groups (Figure 4E).}

\section{Tube formation assay}

The formation of tube-like structures was quantified and the overview of the parameters is shown in figure 5 . Among different parameters of tube formation, four of them was further quantified and normalized with the tube formation in the control group. The four selected parameters were number of nodes, meshes, segments and total length. Results show that hypoxia improved the tube formation and was significantly higher in the HMSC group compared with NMSC. Also, the tube formation was significantly reduced in the NMSC/EC group suggesting that the paracrine effect of EC may have a negative effect on tube formation (Figure 6).

\section{Discussion}

This study shows the effect of a hypoxic microenvironment on the expression of inflammatory and angiogenic cytokines, as well as wound healing and angiogenesis, for MSC cultured alone or in direct co-culture with EC. Hypoxia could alter the expression of inflammatory and vasculogenic cytokines in mono- and co-culture, and the conditioned medium obtained from hypoxia could 
A

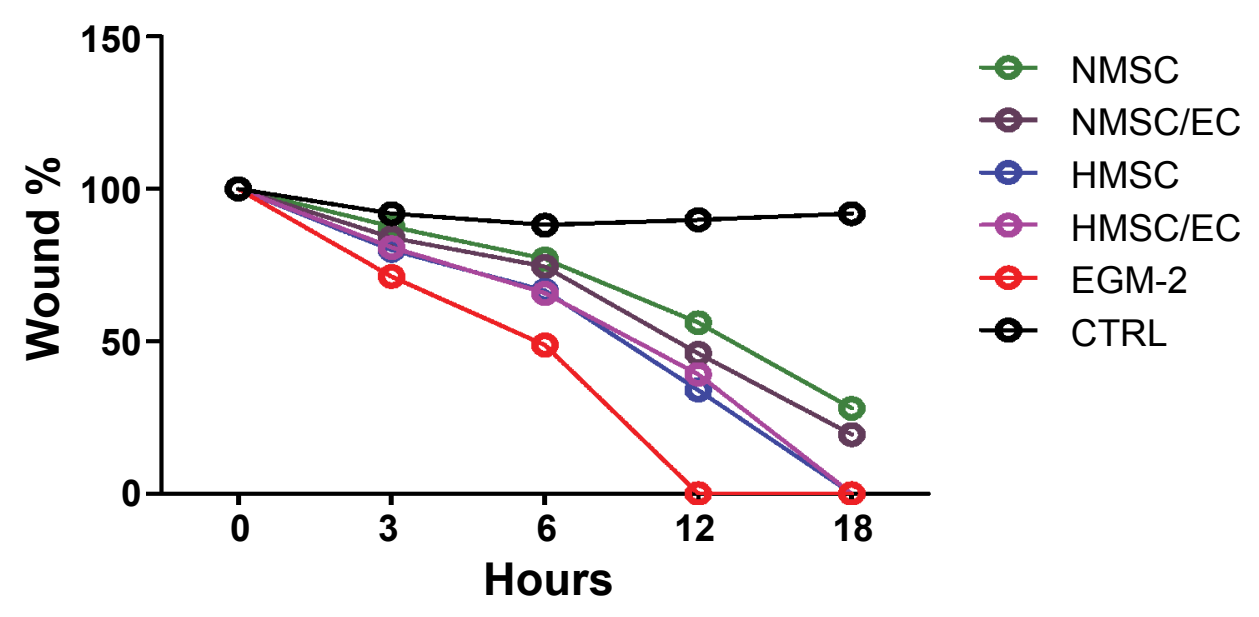

B

\section{C}

3 Hours

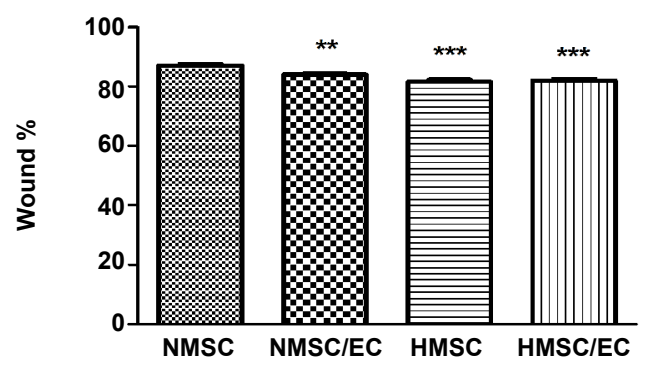

D
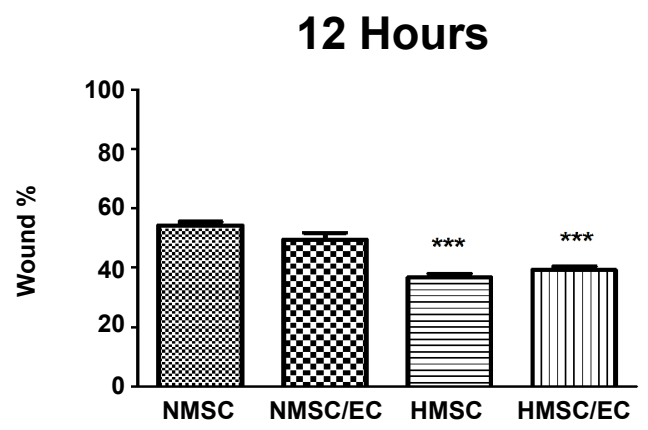

6 Hours

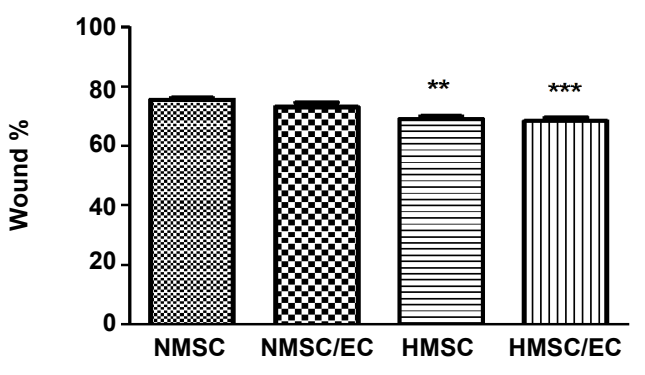

$\mathbf{E}$

\section{Hours}

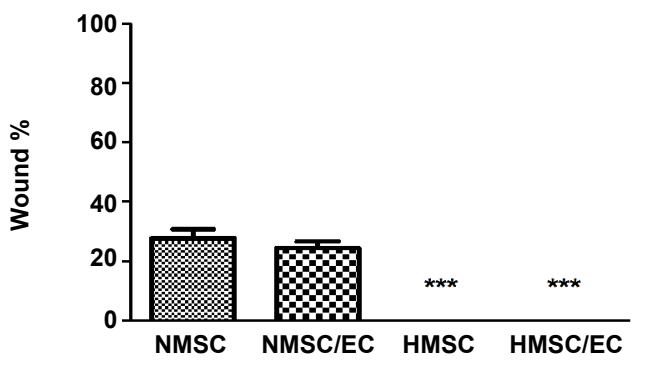

Figure 4: Wound healing assay: The cell migration into the wounded monolayer at different time intervals $(A)$ the percentage of remaining wound in NMSC/EC, HMSC and HMSC/EC compared with NMSC at 3 hours (B) 6 hours (C) 12 hours (D) and 18 hours $(E)$ data are presented as means \pm standard deviation. ${ }^{*}=p<0.05 ;{ }^{* *}=p<0.01 ;{ }^{* *}=p<0.001$ EGM-2 (positive control), CTRL (negative control).

promote wound healing and vessel formation in vitro. The addition of EC increased the immune reactivity of MSC and decreased tube formation. Under normal oxygen tension, transcriptional activity of hypoxia inducible factor-1 (HIF-1) is ubiquitinated and degraded. However, in hypoxic conditions, HIF-1 $\alpha$ gets accumulated and forms a heterodimer with HIF- $1 \beta$ which results in transcription of genes involved in angiogenesis, cell prolifera- tion, cell survival, cell migration and apoptosis [18]. Several studies have demonstrated that the paracrine effects of MSC have regenerative potential [19] and it is known that the conditioned medium (CM) collected from MSC culture in vitro contains different cytokines, chemokines and growth factors [20].

It has been shown that MSC cultured in hypoxia release angiogenic factors such as VEGF-A, MCP-1 and 

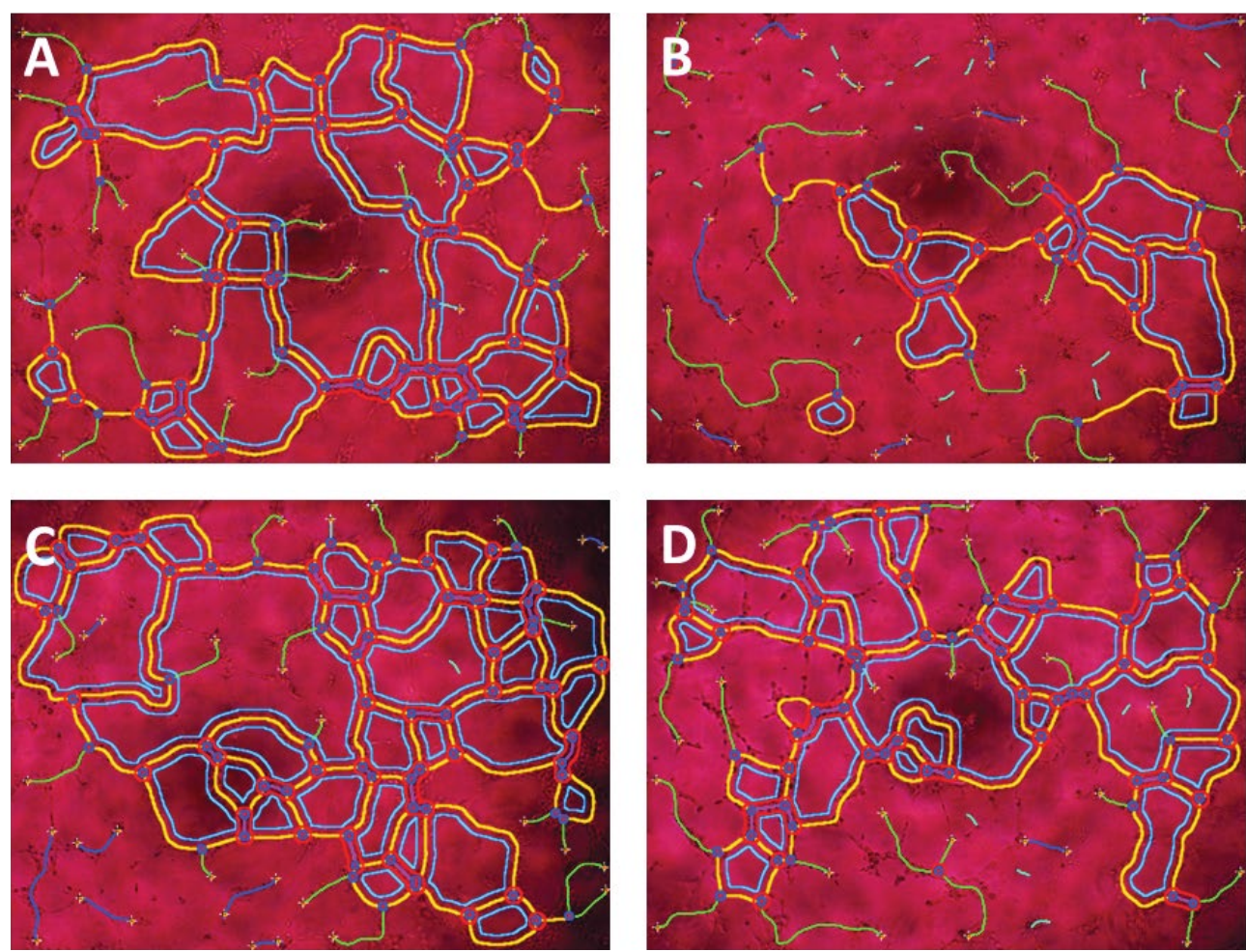

Figure 5: Tube formation assay: The tube formation in different experimental groups at 4x magnification, NMSC (A) NMSC/EC (B) HMSC (C) and HMSC/EC (D) analyzed in ImageJ with angiogenesis plugin.

A

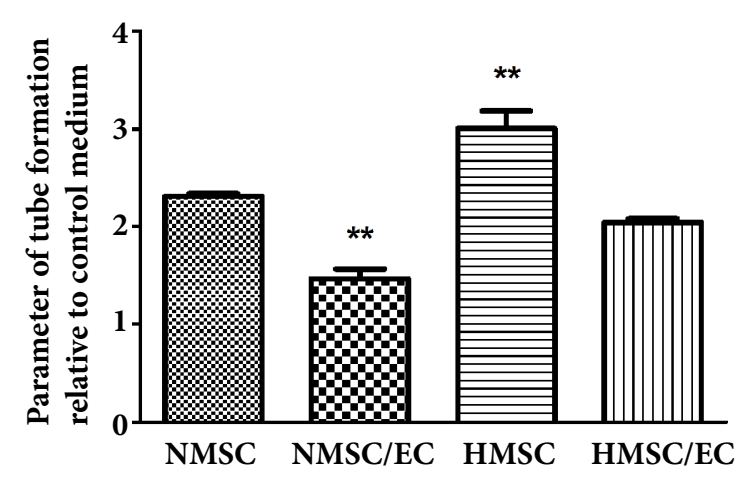

C

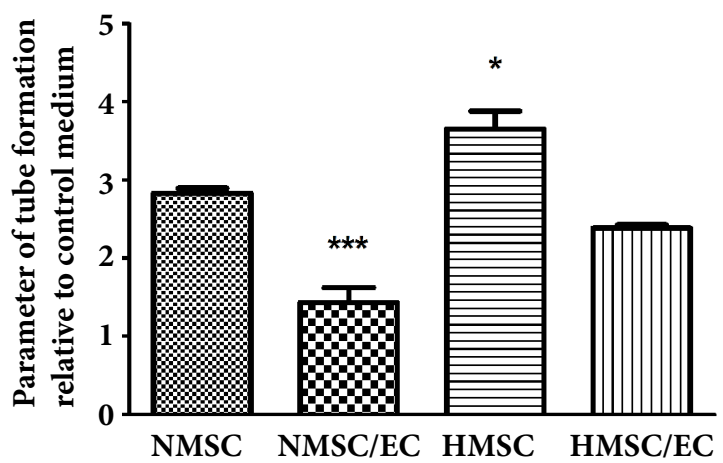

B

MESHES

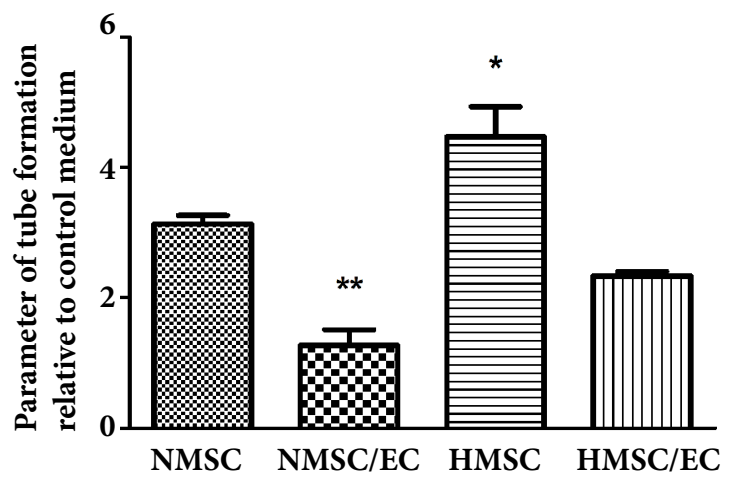

D

TOTAL LENGTH

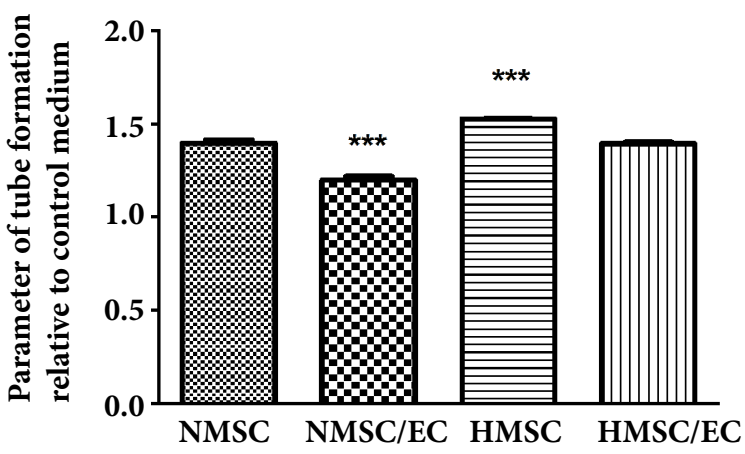

Figure 6: The parameters of tube formation nodes (A) meshes (B) segments (C) and total length (D) in NMSC/EC, HMSC and HMSC/EC groups compared with the NMSC group. Data are presented as means \pm standard deviation. ${ }^{*}=p<0.05 ;{ }^{* *}$ $=p<0.01 ;{ }^{* * *}=p<0.001$. 
angiogenin, which have been detected in the CM [21]. Also the paracrine effect of MSC have been investigated, with results suggesting that MSC grown in normoxia and hypoxia release different soluble factors such as keratinocyte growth factor (KGF), epidermal growth factor (EGF), insulin growth factor (IGF-1), VEGF-A, PDGF, erythropoietin (EPO) and thrombopoietin (TPO) that increase fibroblast, keratinocyte and endothelial cell migration and increase extracellular matrix, which eventually accelerate wound healing [22-24].

We evaluated the release of different cytokines and angiogenic factors released by mono- and co-culture groups under hypoxic and normoxic conditions. Proinflammatory cytokines IL- $1 \beta$, IL- 6 and IL- 8 were upregulated in normoxic co-culture group compared with the NMSC group. However, IL- $1 \beta$ and IL- 8 were downregulated in the HMSC group compared with the NMSC group. Hypoxia preconditioning of MSC could reduce the expression of pro-inflammatory genes, suggesting a potential suppression of the immunoreactivity of MSC. On the contrary, addition of EC increased the shortterm expression of pro-inflammatory cytokines in both the normoxia and hypoxia groups, suggesting increased immunoreactivity from co-culturing the two cell types.

VEGF-Agene- and protein expression was higher in the hypoxic groups. Similar result has been reported in other studies [22] and it is known that MSC express significantly more VEGF-A compared with EC in vitro [25]. This suggests that expression of VEGF-A is induced by hypoxia, independent of the presence of $\mathrm{EC}$, although the paracrine effect of MSC has been shown to induce sprouting of EC via VEGF signalling [26].

ANG-1 has been reported as an angiogenic growth factor responsible for a functional and stable vasculature [27]. We observed that hypoxia reduced the expression of ANG-1, whereas the expression of ANG-2 was increased in both the normoxic and hypoxic co-culture groups. It is known that hypoxia induces the expression of ANG-2 in endothelial cells [28], whereas ANG-1 is down-regulated by hypoxia [29]. Hypoxia has been shown to downregulate the ANG-1/ANG-2 ratio, which could lead to formation of less mature vessels during angiogenesis [30].

Wound healing is a complex process and mediated by the oxygen tension of the surrounding tissue. Hypoxia is a natural process occurring after the formation of a wound. The microenvironment during wound healing attracts and accumulates resident MSC, which migrate to the wounded area. In this hypoxic environment the MSC release paracrine factors that improve healing $[23,24]$. Similarly, we have shown that the conditioned medium obtained from hypoxic cultures improved wound healing in vitro.

The other important parameter during healing and regeneration is vascularization. MSC release increased levels of vasculogenic cytokines, which in turn improve angiogenesis [31]. The hypoxic conditioned medium from MSC and EC were compared separately, which resulted in reduced vessel formation and decreased VEGF-expression in the EC-group compared with MSC [15]. In our study, the vessel formation was reduced and incomplete luminar structures were detected at a higher density in the NMSC/EC-group. Overall, hypoxia increased the formation of vessel-like structures.

The MSC secretomes enhance the formation of vessels. In previous work from our group, pre-vascularized tissueengineered constructs could be generated by co-culturing MSC with EC [16]. Therefore, the combination effect of MSC and EC in this context could lead to improved vascularity of the tissue-engineered constructs. Also, MSC and EC co-culture could generate pre-vascularized constructs in vitro, whereas the paracrine effect of MSC has shown the potential for increased vascular in growth, a feature essential for tissue survival and development. In combination, these elements could result in improved vascularization of tissue-engineered construct and tissue regeneration.

\section{Conclusions}

The present study shows that hypoxia preconditioning altered the expression of inflammatory cytokines and VEGF released by MSC and MSC/EC. The secretome collected from hypoxic culture accelerated wound healing and promoted angiogenesis in vitro.

\section{Acknowledgments}

This study was supported by the VascuBone project, European Union FP7; Grant No. 242175.

\section{Author Contributions}

Conceived and designed the experiments: SBB, TOP, AIB and KM. Performed the experiments: SBB. Analyzed the data: SBB, TOP, AIB and KM. Contributed reagents, materials and analytical tools: TOP, AIB and KM. Wrote the paper: SBB, TOP, AIB and KM. All authors read and approved the final manuscript.

\section{Competing Interests}

The authors declare that they have no competing interests.

\section{References}

1. Watt FM, Hogan BL (2000) Out of Eden: stem cells and their niches. Science 287: 1427-1430.

2. Lee S, Choi E, Cha MJ, Hwang KC (2015) Cell adhesion and long-term survival of transplanted mesenchymal stem cells: a prerequisite for cell therapy. Oxid Med Cell Longev 2015: 632902.

3. Sart S, Ma T, Li Y (2014) Preconditioning stem cells for in vivo delivery. Biores Open Access 3: 137-149.

4. Simon MC, Keith B (2008) The role of oxygen availability in embryonic development and stem cell function. Nat Rev Mol Cell Biol 9: 285-296. 
5. Haque N, Rahman MT, Abu Kasim NH, Alabsi AM (2013) Hypoxic culture conditions as a solution for mesenchymal stem cell based regenerative therapy. ScientificWorldJournal 2013: 632972.

6. Bornes TD, Jomha NM, Mulet-Sierra A, Adesida AB (2015) Hypoxic culture of bone marrow-derived mesenchymal stromal stem cells differentially enhances in vitro chondrogenesis within cell-seeded collagen and hyaluronic acid porous scaffolds. Stem Cell Res Ther 6: 84.

7. Buravkova LB, Andreeva ER, Gogvadze V, Zhivotovsky B (2014) Mesenchymal stem cells and hypoxia: where are we? Mitochondrion 19 Pt A: 105-112.

8. Janeczek Portalska K, Leferink A, Groen N, Fernandes H, Moroni L, et al. (2012) Endothelial differentiation of mesenchymal stromal cells. PLoS One 7: 46842.

9. Oswald J, Boxberger S, Jorgensen B, Feldmann S, Ehninger $G$, et al. (2004) Mesenchymal stem cells can be differentiated into endothelial cells in vitro. Stem Cells 22 377-384.

10. Zeng X, Zeng YS, Ma YH, Lu LY, Du BL, et al. (2011) Bone marrow mesenchymal stem cells in a three-dimensional gelatin sponge scaffold attenuate inflammation, promote angiogenesis, and reduce cavity formation in experimental spinal cord injury. Cell Transplant 20: 1881-1899.

11. Koike N, Fukumura D, Gralla O, Au P, Schechner JS, et al. (2004) Tissue engineering: creation of long-lasting blood vessels. Nature 428: 138-139.

12. Laschke MW, Menger MD (2012) Vascularization in tissue engineering: angiogenesis versus inosculation. Eur Surg Res 48: 85-92.

13. Auger FA, Gibot L, Lacroix D (2013) The pivotal role of vascularization in tissue engineering. Annu Rev Biomed Eng 15: $177-200$.

14. Bader AM, Klose K, Bieback K, Korinth D, Schneider M, et al. (2015) Hypoxic Preconditioning Increases Survival and Pro-Angiogenic Capacity of Human Cord Blood Mesenchymal Stromal Cells In Vitro. PLoS One 10: e0138477.

15. Zhang B, Yang S, Zhang Y, Sun Z, Xu W, et al. (2012) Co-culture of mesenchymal stem cells with umbilical vein endothelial cells under hypoxic condition. J Huazhong Univ Sci Technolog Med Sci 32: 173-180.

16. Pedersen TO, Blois AL, Xing Z, Xue Y, Sun Y, et al. (2013) Endothelial microvascular networks affect gene-expression profiles and osteogenic potential of tissue-engineered constructs. Stem Cell Res Ther 4: 52.

17. Carpentier G (2012) ImageJ contribution: Angiogenesis Analyzer. Image J News.

18. Semenza GL (2003) Targeting HIF-1 for cancer therapy. Nat Rev Cancer 3: 721-732.

19. Burdon TJ, Paul A, Noiseux N, Prakash S, Shum-Tim D (2011) Bone marrow stem cell derived paracrine factors for regenerative medicine: current perspectives and therapeu- tic potential. Bone Marrow Res 2011: 207326.

20. Kinnaird T, Stabile E, Burnett MS, Shou M, Lee CW, et al. (2004) Local delivery of marrow-derived stromal cells augments collateral perfusion through paracrine mechanisms. Circulation 109: 1543-1549.

21. Hung SC, Pochampally RR, Chen SC, Hsu SC, Prockop DJ (2007) Angiogenic effects of human multipotent stromal cell conditioned medium activate the PI3K-Akt pathway in hypoxic endothelial cells to inhibit apoptosis, increase survival, and stimulate angiogenesis. Stem Cells 25: 2363-2370.

22. Chen L, Tredget EE, Wu PY, Wu Y (2008) Paracrine factors of mesenchymal stem cells recruit macrophages and endothelial lineage cells and enhance wound healing. PLoS One 3: e1886.

23. Lee DE, Ayoub N, Agrawal DK (2016) Mesenchymal stem cells and cutaneous wound healing: novel methods to increase cell delivery and therapeutic efficacy. Stem Cell Res Ther 7: 37 .

24. Chen L, Xu Y, Zhao J, Zhang Z, Yang R (2014) Conditioned medium from hypoxic bone marrow-derived mesenchymal stem cells enhances wound healing in mice. PLoS One 9: e96161.

25. Pedersen TO, Blois AL, Xue $Y$, Xing Z, Sun $Y$ et al. (2014) Mesenchymal stem cells induce endothelial cell quiescence and promote capillary formation. Stem Cell Res Ther 5: 23.

26. Beckermann BM, Kallifatidis G, Groth A, Frommhold D, Apel A, et al. VEGF expression by mesenchymal stem cells contributes to angiogenesis in pancreatic carcinoma. $\mathrm{Br} \mathrm{J}$ Cancer 99: 622-631.

27. Suri C, Jones PF, Patan S, Bartunkova S, Maisonpierre PC, et al. (1996) Requisite role of angiopoietin-1, a ligand for the TIE2 receptor, during embryonic angiogenesis. Cell 87: 1171-1180.

28. Simon MP, Tournaire R, Pouyssegur J (2008) The angiopoietin-2 gene of endothelial cells is up-regulated in hypoxia by a HIF binding site located in its first intron and by the central factors GATA-2 and Ets-1. J Cell Physiol 217: 809-818.

29. Enholm B, Paavonen K, Ristimaki A, Kumar V, Gunji Y, et al. (1997) Comparison of VEGF, VEGF-B, VEGF-C and Ang-1 mRNA regulation by serum, growth factors, oncoproteins and hypoxia. Oncogene 14: 2475-2483.

30. Lund EL, Høg A, Olsen MW, Hansen LT, Engelholm SA, et al. (2004) Differential regulation of VEGF, HIF1 $\alpha$ and angiopoietin-1, -2 and -4 by hypoxia and ionizing radiation in human glioblastoma. Int J Cancer 108: 833-838.

31. Boomsma RA, Geenen DL (2012) Mesenchymal stem cells secrete multiple cytokines that promote angiogenesis and have contrasting effects on chemotaxis and apoptosis. PLoS One 7: e35685. 